\section{Mast Cell Granules}

By B. UvNäs (Department of Pharmacology, Karolinska Institutet, Stockholm)

\section{The Storage of Transmitters in the Central Nervous System}

By V. P. Whittaker (Department of Biochemistry, Cambridge)

There is increasing evidence that nerve impulses are relayed from one nerve cell to another, centrally as well as peripherally, by the release, from the presynaptic nerve terminals, of chemical transmitter substances. These include acetylcholine, a group of monoamines (noradrenaline, dopamine, 5hydroxytryptamine) and, less certainly, several amino acids, notably $\gamma$-aminobutyrate, glycine, glutamate and aspartate. Many centrally acting drugs are chemical analogues of some of these substances, especially acetylcholine and the monoamines, and it is a reasonable hypothesis that they modify brain function by interfering, in one way or another, with the processes of synthesis, storage, release and post-synaptic action of chemical transmitters.

The study of these processes at the molecular level has been aided by the discovery that when brain tissue is homogenized under carefully controlled conditions in iso-osmotic media, presynaptic nerve terminals are broken off from their axons and post-synaptic attachments to form sealed bags (synaptosomes) that retain the morphology and transmitter content of the original terminals (reviewed by Whittaker, 1965, 1969a,b). The equilibrium density of synaptosomes in sucrose density gradients is greater than that of myelin but less than that of free mitochondria or aminestorage granules from other tissues; this enables them to be separated from the other two main constituents of crude brain mitochondrial fractions and explains why bound noradrenaline and 5 . hydroxytryptamine in brain preparations subjected to density-gradient centrifugation are recovered from less dense regions of the gradient than in corresponding experiments with adrenal medulla or intestine.

For many purposes, a fraction enriched in synaptosomes (e.g. fraction $B$, Gray \& Whittaker, 1962) or even a crude fraction (fraction $P_{2}$ ) containing a mixture of synaptosomes, free mitochondria, myelin and glial fragments is adequate, but studies of the lipid and protein composition of synaptic membranes will increasingly require more homogeneous fractions in greater quantities. These can be obtained by centrifugal separations in sucrose density gradients with swing bucket (Fonnum,
1968; Whittaker, 1968a) or zonal rotors (E. K. Miller \& V.P. Whittaker, unpublished observations).

Recent studies in my Laboratory have been mainly concerned with the organization of the cholinergic terminal. Earlier work showed that bound acetylcholine in brain homogenates (i.e. acetylcholine immune to the action of cholinesterases and pharmacologically inactive) represented about $70-75 \%$ of the total brain acetylcholine; the remainder disappeared unless homogenization was carried out in the presence of a cholinesterase inhibitor, and was then recovered in free form in the high-speed supernatant fraction. Bound acetylcholine is localized in synaptosomes; about half of it (the 'labile-bound' fraction; Whittaker, 1959) represents acetylcholine sequestered within the synaptosomal cytoplasm and the remainder (the 'stable-bound ' fraction) is associated with the synaptic vesicles, the characteristic organelles, about $500 \AA$ in diameter, of the presynaptic terminal cytoplasm. Since the vesicles represent only about $3-4 \%$ of the total volume of the synaptosome (Clementi, Whittaker \& Sheridan, 1966), the concentration of the acetylcholine in the vesicles is 25-30 times that in the cytoplasm and is probably close to being iso-osmotic with cytoplasm (Whittaker \& Sheridan, 1965). Thus the vesicles can be regarded as being the ultimate storage site of the transmitter in the terminal. However, $\left[{ }^{3} \mathrm{H}\right]$ cholineincorporation studies (L. W. Chakrin and V. P. Whittaker, unpublished work) show that cytoplasmic acetylcholine is not an artefact caused by leakage from the vesicles during isolation, as has sometimes been suggested. When $\left[{ }^{3} \mathrm{H}\right]$ choline is injected into the brain substance of anaesthetized animals (cat, guinea pig, rabbit) and synaptosomes and synaptic vesicles subsequently isolated by the methods previously developed (Gray \& Whittaker, 1962; Whittaker, Michaelson \& Kirkland, 1964), the specific radioactivity of the vesicular acetylcholine is about one-third of that of the cytoplasmic acetylcholine $1 \mathrm{hr}$. after injection. In earlier experiments in vitro with isolated synaptosomes, March. banks (1968) found negligible labelling of vesicles under conditions which permitted considerable labelling of the cytoplasmic compartment, and it is clear that even under the more physiological conditions of labelling in vivo the two compartments retain their separate identities.

In contrast with acetylcholine, the enzyme synthesizing acetylcholine (choline acetyltransferase) is confined to one synaptosomal compartment, the soluble cytoplasmic (Whittaker et al. 1964). Evidence to the contrary (McCaman, Arnaiz \& De Robertis, 1965; Saelens \& Potter, 1966) is explained by non-specific adsorption of the enzyme on to fragments of external membrane at the low ionic strengths necessary for the hypo- 
osmotic rupture of synaptosomes and the subsequent separation of their component organelles, together with failure to separate vesicles adequately from fragments of external membranes (Fonnum, 1967; Potter, 1968). Evidently acetylcholine is synthesized in the cytoplasm and subsequently transferred to the vesicles by an uptake mechanism that has so far not proved reproducible in isolated synaptosomes or synaptic vesicles (Marchbanks, 1968).

Acetylcholinesterase, the third component of the cholinergic neurone, is known from histochemical studies (Lewis \& Shute, 1966) to be distributed throughout the length of such neurones: in the cell body it is present in the lumen of the endoplasmic reticulum; in the axon, on the plasma membrane, and at the terminal, on the external presynaptic membrane. The resolution of the histochemical method is not sufficiently good to determine whether the enzyme is on the inside or on the outside of the membrane, but functional studies with isolated synaptosomes suggest that the latter is true. Thus the rate of destruction of free cyto. plasmic acetylcholine appears to be diffusionlimited and is very much slower than that of a similar concentration of externally applied acetylcholine. The subcellular localization of the enzyme is consistent with its histochemical localization; it is present in highest specific activity in fractions rich in microsomes and external synaptosome membranes, but is absent from synaptic vesicles, soluble cytoplasm or intraterminal mitochondria (Whittaker et al. 1964; Wiegandt, 1967).

Synaptosomes are metabolically active when incubated at $25-30^{\circ}$ in saline media fortified with coenzymes, and under these conditions take up choline by a $\mathrm{Na}^{+}$-dependent, hemicholinium. sensitive mechanism (Potter, 1968; R. M. Marchbanks, unpublished work). They appear to be able to synthesize protein in vitro (Morgan \& Austin, 1968) and provide samples of terminal axoplasm in studies of axonal flow (Barondes, 1966, 1968): At $0-4^{\circ}$, their metabolism is arrested and their internal composition can be modified by equilibration with different suspension media (Marchbanks, 1967). This enables the passive permeability of their external membranes to be studied. Synaptosomes can thus be regarded as model neurones or miniature cells in studies of plasma membrane function (Whittaker, 1968b).

Much of the recent work from this Laboratory has been supported by the Medical and Science Research Councils.

Barondes, S. H. (1966). J. Neurochem. 13, 721.

Barondes, S. H. (1968). J. Neurochem. 15, 343.

Clementi, F., Whittaker, V. P. \& Sheridan, M. N. (1966). Z. Zellforsch. 72, 126.

Fonnum, F. (1967). Biochem. J. 108, 262.

Fonnum, F. (1968). Biochem. J. 106, 401.

Gray, E. G. \& Whittaker, V. P. (1962). J. Anat., Lond., 96, 79.

Lewis, P. R. \& Shute, C. C. D. (1966). J. Cell Sci. 1, 381.

Marchbanks, R. M. (1967). Biochem. J. 104, 148.

Marchbanks, R. M. (1968). Biochem. J. 106, 87.

McCaman, R. E., Arnaiz, G. R. de L. \& De Robertis, E. (1965). J. Neurochem. 12, 927.

Morgan, I. G. \& Austin, L. (1968). J. Neurochem. 15, 41.

Potter, L. T. (1968). In The Interaction of Drugs and Sub. cellular Components in Animal Cells, p. 293. Ed. by Campbell, P. N. London: J. \& A. Churchill Ltd.

Saelens, J. K. \& Potter, L. T. (1966). Fed. Proc. 25, 451.

Whittaker, V. P. (1959). Biochem. J. 72, 694.

Whittaker, V. P. (1965). Progr. Biophys. mol. Biol. 15, 39.

Whittaker, V. P. (1968a). Biochem. J. 106, 492.

Whittaker, V. P. (1968b). Brit. med. Bull. 24, 1.

Whittaker, V. P. (1969a). In Structure and Function of Nervous Tissue, vol. 2, p. 1. Ed. by Bourne, G. H. New York: Academic Press Inc.

Whittaker, V. P. (1969b). In Handbook of Neurochemistry, vol. 1. Ed. by Lajtha, A. New York: Plenum Press.

Whittaker, V. P. \& Sheridan, M. N. (1965). J. Neurochem. $12,363$.

Whittaker, V. P., Michaelson, I. A. \& Kirkland, R. J. A. (1964). Biochem. J. 80, 293.

Wiegandt, H. (1967). J. Neurochem. 14, 671 .

\section{COMMUNICATIONS}

\section{Studies on the Conformation of Polygalact- uronic Acid in Solution}

By R. W. Stoddart* and K. F. Tipton (Department of Biochemistry, University of Cambridge)

A titration curve of polygalacturonic acid gives a single $\mathrm{p} K$ in the acid region, corresponding to the

\footnotetext{
* Present address: Medical Research Council Molecular Pharmacology Research Unit, Old Press Site, Mill Lane, Cambridge.
}

ionization of the carboxyl groups. Changes in a number of the physical properties of the polymer parallel this titration curve. When the dye ruthenium red is bound to polygalacturonic acid there is a shift and an increase of its visible absorption spectrum, and this metachromic shift has been shown to be $\mathrm{pH}$-sensitive. As the $\mathrm{pH}$ is lowered the spectral shift progressively diminishes towards zero and the enhanced absorption at $\mathrm{pH} 6.5$ changes to a hypochromic effect at low $\mathrm{pH}$ values. Both these changes give titration curves resembling the acid titration curve of polygalacturonic acid. 